\title{
Nanofocus diamond X-ray windows: Thermal modeling of nano-sized heat source systems
}

\author{
Colin Delfaure ${ }^{\mathrm{a}, *}$, Jean Paul Mazellier ${ }^{\mathrm{b}}$, Nicolas Tranchant ${ }^{\mathrm{a}}$, Philippe Bergonzo ${ }^{\mathrm{a}}$, Pascal Ponard ${ }^{\mathrm{c}}$, Samuel Saada $^{\mathrm{a}}$ \\ a CEA, LIST, Diamond Sensors Laboratory, Gif-sur-Yvette 91191, France \\ b Thales Research and Technology, Route Départementale 128, Palaiseau 91767, France \\ c Thales Electron Devices, ZI de Vongy, 74202 Thonon-les-Bains, France
}

\section{A R T I C L E I N F O}

\section{Article history:}

Received 7 May 2015

Received in revised form 31 August 2015

Accepted 25 September 2015

Available online 28 September 2015

\section{Keywords:}

Thermal model

Diamond window

Nanofocus

Microfocus

X-ray tube

Thermal management

\begin{abstract}
A B S T R A C T
An analytical thermal model is proposed to study heat transfers occurring at high power density in X-ray tubes with micron to submicron sized source. The use of a simple analytical approach instead of a complex numerical simulation allows readily modeling of more and more challenging systems such as multi-source X-ray tubes. By significantly reducing the computing time, it enables a wider parameter range evaluation for engineering phase. We focused our work on tubes integrating a transmission window that is mainly edge-cooled. Our approach can be generally used in cylindrical lateral heat spreaders with distributed small sized source systems. The model enables an efficient estimation of temperature distributions for a large range of parameters and source designs and is, for instance, wellsuited to described nanosized heat source systems. It is developed from an electrostatic analogy with the point charge particle model and uses of a series of virtual sources. A multiscale resolution of the heat equation is proposed, hence providing the temperature distribution at any point within the whole system. Moreover, the non-linearity of equations caused by temperature-dependent thermal conductivities is solved by using Kirchhoff's transformation, giving a more realistic approach of heat conduction in diamond X-ray windows, where temperature in excess of $1000^{\circ} \mathrm{C}$ can be encountered. The influence of convective and radiative transfers has been discussed and the physical accuracy of the predicted temperature is controlled by adjusting the number of virtual sources of the model.
\end{abstract}

(c) 2015 Elsevier B.V. All rights reserved.

\section{Introduction}

Recent developments in X-ray imaging systems have led to innovative X-ray tube architectures for high resolution imaging based on submicron spot size [1-3]. For this purpose, transmission X-ray tubes are particularly well-suited. They are based on a window-target stack, ensuring the mechanical separation between the vacuum (inside the tube) and the external environment (typically ambient air). In the tube, a highly energetic focused electron beam impacts the target made of a high atomic number metal such as gold or tungsten. A part of the impinging electron beam energy is converted into X-rays through two main processes: Bremsstrahlung and edge transition processes (i.e. core electrons of the target atoms are ejected and X-ray is emitted when relaxation process occurs). Generally speaking, the target thickness results from a compromise between electron penetration depth and X-ray self-absorption. As a thumb of rule, the typical thickness ranges from 3 to $15 \mu \mathrm{m}$. As this thickness is not compatible with a 1 bar differential pressure resistance, a support material, denoted as window, has to be used to support the target and ensure vacuum sealing of the tube. Another point concerns the low conversion efficiency from

\footnotetext{
* Corresponding author.

E-mail address: colin.delfaure@cea.fr (C. Delfaure).
}

electron energy to X-ray (about 1\%). The remaining energy (99\%) is converted into heat in the target material. Again, the target small thickness impedes dramatically its thermal resistance. As a result, the window also ensures the role of heat spreader. Taking into account these considerations, the window material is chosen to ensure two functions: it must exhibit an appropriate mechanical strength to be vacuum tight and must feature the highest thermal conductivity in order to lower the target temperature under operation. In addition, it must present a good X-ray transparency in order to reduce the flux loss. The standard material used for transmission X-ray tubes is beryllium because it fulfills these requirements for common applications where typical power densities range from $10^{10} \mathrm{~W} / \mathrm{m}^{2}$ to $2.10^{11} \mathrm{~W} / \mathrm{m}^{2}$. Its thermal conductivity is around $200 \mathrm{~W} /(\mathrm{m} \cdot \mathrm{K})$ at room temperature [4].

But application requirements impose increased spatial resolution associated with punctual X-ray sources. This can be approached by reducing the electron beam spot size on the target to submicron sizes. Typical associated heat densities can reach values as high as $2.10^{14} \mathrm{~W} / \mathrm{m}^{2}$. As a consequence the downscaling of the X-ray source size shed a new light on thermal issues occurring in the target/window system subjected to huge local heat densities [3]. In this case, beryllium exhibits limitations in dealing with such heat densities. On the contrary, diamond is a good candidate for such applications as it is composed only of carbon (low Z material with high X-ray transparency) and it has the highest known 
thermal conductivity of all bulk materials (up to $2000 \mathrm{~W} /(\mathrm{m} / \mathrm{K}$ ) for single crystal diamond at room temperature) [5-7,35]. It has recently emerged as viable solution for transmission type X-ray generators with higher thermal performances.

The harsh thermal constraints have to be taken into account at the earliest stage of system development. An efficient tool is requested to anticipate this particular point. The common finite element modeling (FEM) approach is known to be efficient but particularly resource consuming when multiscale simulations have to be implemented. This is the case here where nanoscale heat sources of tremendous power densities have to be considered on centimeter scale systems. Analytical models would be advantageously used in the present case and would allow fast system evaluation. In this work, we are interested in computing the temperature distribution within a high $\mathrm{Z}$ target (tungsten, copper, gold) and its supporting membrane (diamond or beryllium) used as the X-ray window. This target is hit by a high energy electron micro/nanometric beam that causes the system to locally heat up to temperatures high enough for inducing target melting (about $4000 \mathrm{~K}$ ) [8]. We report the derivation of an analytical model that can be used to determine the temperature at any point of the target/windows system. This analytical model includes the dominant heat transport phenomenon occurring in this configuration. We systematically compared the model results with simulations. Furthermore, we compared the results obtained with our description to experimental data, in particular concerning target melting issues.

\section{Modeling steady-state temperature of edge-cooled window}

We considered a general problem based on a cylindrical window (as depicted in Fig. 1), of radius $\mathrm{R}$ and height $\mathrm{H}$, which is the most common X-ray transmission tube window geometry. The membrane is considered to be edge-cooled by conduction only. The periphery of the cylinder is kept at a constant temperature by a perfect thermostat. A point heat source, dissipating a total power $Q$ is placed at the center of the bottom face of the cylinder. The cylinder is made of perfect single crystal diamond with a constant thermal conductivity $\lambda$ (no temperature dependence is taken into account at this stage).

Because of transparency requirements in X-ray transmission windows, the thickness of the target has to lay in the micrometer range for X-ray production and those of the window in tens to hundreds of micrometers to withstand differential pressure constraints. When one compares these dimensions to the lateral extension of the window (typically some millimeters), it is no longer possible to assume a constant temperature on the top surface (window-environment interface, see Fig. 1) as it has often been proposed in other models [9-12]. Our model takes this geometry constraint into account to accurately simulate the field temperature in the system.

\subsection{Model development}

We first consider a bulk system (homogeneous material with thermal conductivity $\lambda$ independent of temperature) constituted by a cylinder of radius $\mathrm{R}$ and thickness $\mathrm{H}$ (cf. Fig. 2). A point heat source, of total power $\mathrm{Q}$ is placed at the center of the $\mathrm{z}=0$ planar face. The membrane lateral edge is kept at temperature $T_{t h}$ by a perfect thermostat. No heat transfers on the top and bottom faces of the cylinder are considered here. For computing the temperature field in this system, an analogous system has been studied (see Fig. 2). It is constituted of an infinite cylinder of radius $\mathrm{R}$, of the same material as the membrane described above. An infinite series of point sources is placed on the axial symmetry axis of the infinite cylinder. Each point source dissipates a total power equal to $2 \mathrm{Q}$. The sources are spaced by a distance $2 \mathrm{H}$. The lateral edge of the infinite cylinder is kept at temperature $T_{t h}$ by a perfect thermostat.

By symmetry reasons, we can define two families of particular planes in this system:

- Planes perpendicular to the cylinder central axis and containing a heat point source, defining a family of planes noted as $\mathrm{P}_{\mathrm{b}, \mathrm{n}}$

- Planes perpendicular to the cylinder central axis and equidistant from two adjacent heat sources, defining a family of planes noted as $\mathrm{P}_{\mathrm{t}, \mathrm{n}}$.

It is obvious that there is no heat transfer perpendicular to these two sets of families occurring because of their symmetry properties. Furthermore, it is clear that the temperature field observed between planes $\left\{\mathrm{P}_{\mathrm{b}, \mathrm{n}} ; \mathrm{P}_{\mathrm{t}, \mathrm{n}}\right\}$ (or $\left\{\mathrm{P}_{\mathrm{b}, \mathrm{n}} ; \mathrm{P}_{\mathrm{t}, \mathrm{n}-1}\right\}$ ) is homologous to the membrane field temperature we are interested in. The $P_{b, n}$ plane is equivalent to the bottom face of the window (where the source is located) and the $P_{t, n}$ plane being equivalent to the top face of the window.

An analogy with the Poisson's equation for electrostatic potential around a charged point particle in an infinite media, gives the expression of temperature around a single source (within a constant factor).

$T(\mathrm{r}, \mathrm{z})=\frac{2 \mathrm{Q}}{4 \pi \lambda} \frac{1}{\sqrt{\mathrm{r}^{2}+\mathrm{z}^{2}}}$

where $Q$ is the power of the heat source and $\lambda$ the thermal conductivity of the window. The geometrical parameters $r$ and $z$ are represented in Fig. 2 together with the infinite cylinder model and with an infinite series of virtual sources along the $\mathrm{z}$ axis. We propose to index the sources by considering the source at $\mathrm{z}=0$ to be indexed as 0 , index increasing with $z$ value. The distance $d_{n}(r, z)$ between the $n$th source and a point of coordinates $(r, z)$ is expressed as

$\mathrm{d}_{\mathrm{n}}(\mathrm{r}, \mathrm{z})=\sqrt{\mathrm{r}^{2}+(2 n \mathrm{H}-\mathrm{z})^{2}}=\mathrm{H} \sqrt{(\mathrm{r} / \mathrm{H})^{2}+(2 n-\mathrm{z} / \mathrm{H})^{2}}$.

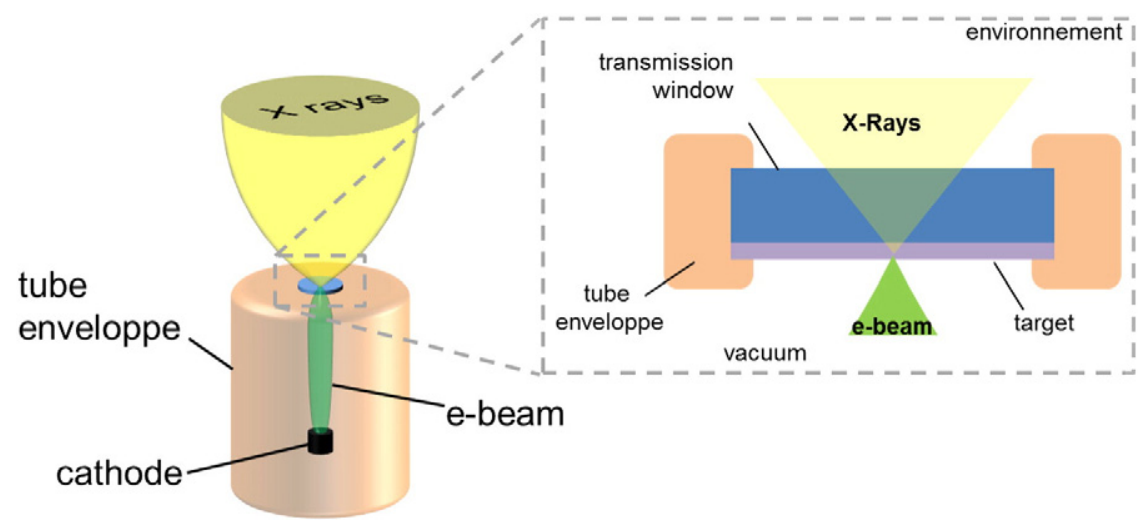

Fig. 1. Sketch of an X-ray tube in transmission configuration. 


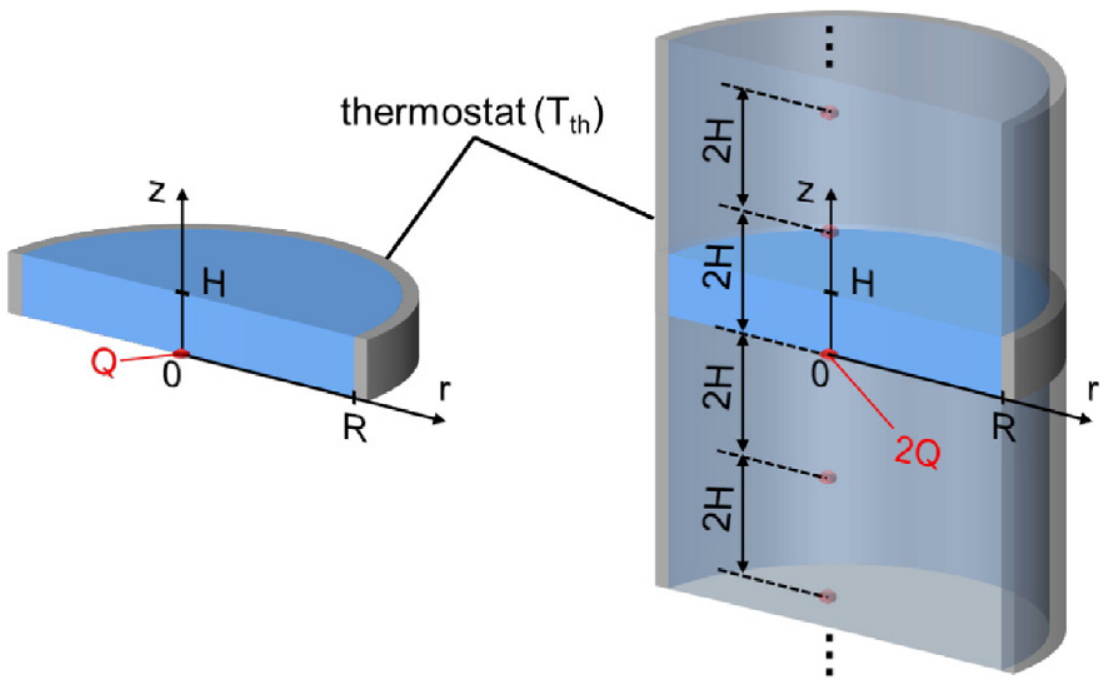

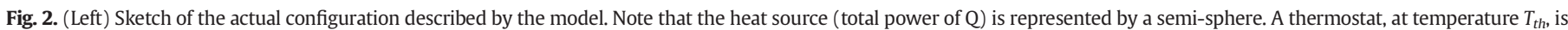

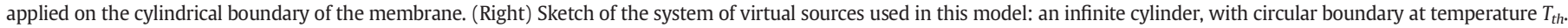

Using expressions (1) and (2), we can write the sum of the contribution of each individual point source as a linear superposition of each contribution.

$T(\mathrm{r}, \mathrm{z})=\frac{\mathrm{Q}}{2 \pi \mathrm{H} \lambda} \sum_{n=-\infty}^{+\infty} \frac{1}{\sqrt{(\mathrm{r} / \mathrm{H})^{2}+(2 n-\mathrm{z} / \mathrm{H})^{2}}}$

Because there are an infinite number of heat sources in the $\mathrm{z}$ direction, this expression tends to diverge. Similar problems have been addressed for one-dimensional electrostatic systems by considering the electrical potential difference between any point in the system and a point where the potential is determined $[13,14]$. By analogy, the temperature thermostat at the lateral edge substitutes the reference potential. The temperature difference between any point $(r, z)$ in the membrane and a point $\left(\mathrm{R}, \mathrm{Z}^{\prime}\right)$ of the edge (kept at thermostat temperature $T_{\text {th }}$ ) can be expressed from Eq. (3):

$\Delta T(\mathrm{r}, \mathrm{z})=\frac{\mathrm{Q}}{2 \pi \mathrm{H} \lambda} \sum_{\mathrm{n}=-\infty}^{+\infty}\left\{\frac{1}{\sqrt{\frac{\mathrm{r}^{2}}{\mathrm{H}^{2}}+\left(2 n-\frac{\mathrm{z}}{\mathrm{H}}\right)^{2}}}-\frac{1}{\sqrt{\frac{\mathrm{R}^{2}}{\mathrm{H}^{2}}+\left(2 n-\frac{\mathrm{z}^{\prime}}{\mathrm{H}}\right)^{2}}}\right\}$

The constant temperature along the lateral edge of the window implies that any value of $z^{\prime}$ can be used in the previous expression. For the sake of clarity, $z^{\prime}$ will be taken equals to 0 in the following developments.

Splitting the series between negatives and positives $\mathrm{n}$ indexes and bounding both series with an integral comparison test lead to prove the convergence of the global series of Eq. (4) (see details in Appendix A). The limit of this series is referred as $\xi\left(r^{*}, R^{*}, z^{*}\right)$ and the temperature rise in the $\mathrm{X}$-ray window is written as:

$\Delta T(\mathrm{r}, \mathrm{z})=\frac{\mathrm{Q}}{2 \pi \mathrm{H} \lambda} \xi\left(\mathrm{r}^{*}, \mathrm{R}^{*}, \mathrm{z}^{*}\right)$.

We introduce here the reduced variables defined as $x^{*}=x / H, x$ being a geometrical dimension. Expression 5 gives the temperature distribution in a membrane made of a single material. When $r \gg H$, the upper and lower bounds used to prove the convergence of the series are the same (see Appendix A), thus providing the exact solution for $\xi$. The temperature is then given by the simple form of Eq. (6).

$\Delta T(\mathrm{r} \gg \mathrm{H}, \mathrm{z})=\frac{\mathrm{Q}}{2 \pi \mathrm{H} \lambda} \log \left(\frac{\mathrm{R}}{\mathrm{r}}\right)$

This solution matches the expression of temperature at a distance $\mathrm{r}$ around an infinite linear source ( $\mathrm{z}$ axis) with a linear heat source of $2 \mathrm{Q} / \mathrm{H}$ as can be expected. In the general case, the expression of $\xi\left(r^{*}, R^{*}, z^{*}\right)$ cannot be simplified but in order to fasten its computation, we define a truncation of the series to $2 \mathrm{P}+1$ sources and this truncated function can be tabulated for the normalized set of parameters. A sufficient number of sources must be used to ensure a correct precision of the model. As a matter of fact, the rest of the series $\xi$ after the $(2 \mathrm{P}+1)^{\text {th }}$ term is directly proportional to the error $\delta T$ made on the temperature by truncating this expression. The accuracy criterion chosen here is a tenth of a degree and the minimal number of source verifying this criterion can easily be calculated (see Appendix B). This minimal number of heat sources is proportional to $\mathrm{Q} / \lambda$ but is also dependent

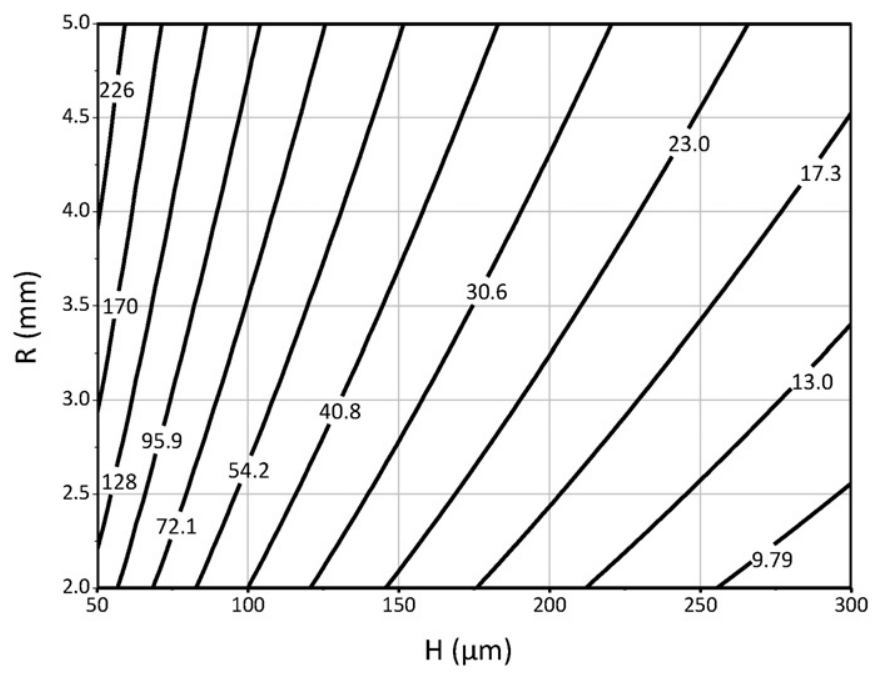

Fig. 3. Contour plot of the minimal number of source to ensure accuracy below $0.1 \mathrm{~K}$ on the modeled temperature as a function of the geometrical dimension of the window. 


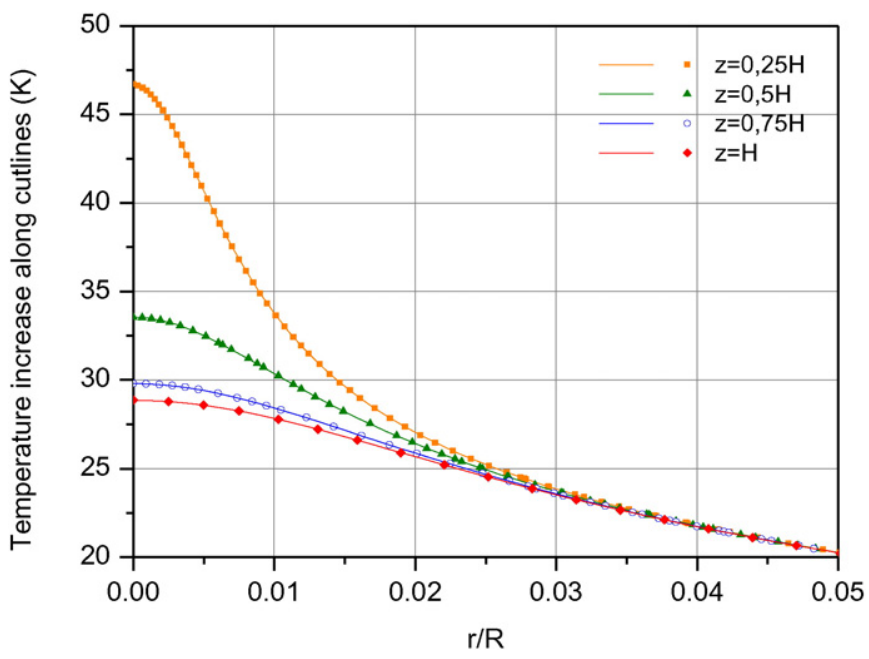

Fig. 4. Temperature increase along cut lines perpendicular to the cylinder axis for a diamond membrane of thickness $\mathrm{H}=100 \mu \mathrm{m}$ and radius $\mathrm{R}=50 \mathrm{H} .125$ sources are needed to get accurate results (error below the tenth of degree). A heat source of $8.5 \mathrm{~W}$ is used and the thermal conductivity of the window is set to $2000 \mathrm{~W} /(\mathrm{m} \cdot \mathrm{K})$. The markers refer to finite element simulations while solid lines refer to our model results.

on the geometrical parameters $\mathrm{R}$ and $\mathrm{H}$ (see details in Appendix B). It is convenient to represent it versus the geometrical dimensions of the membranes on an abacus as presented on Fig. 3. Once this abacus is used to determine the number of virtual sources needed, the validity of the truncation can be checked on Fig. 4 by comparing the results
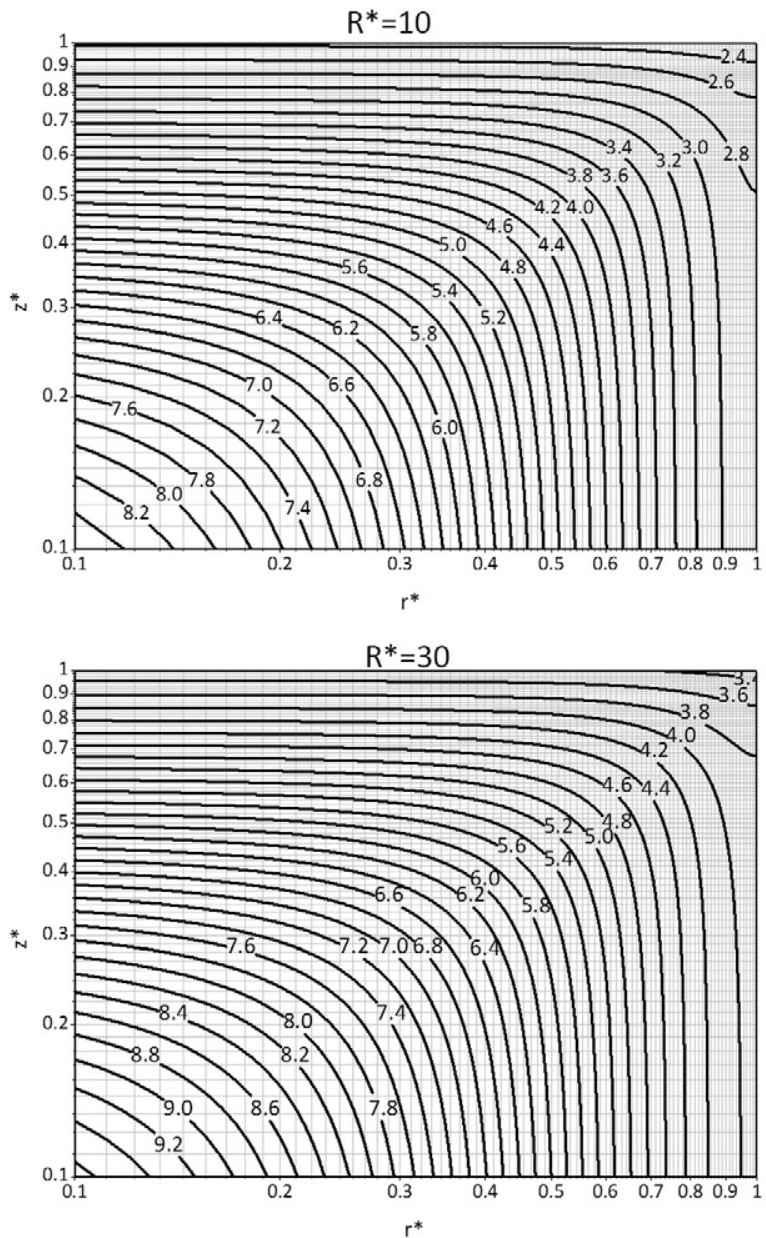

obtained with our model to a FEM simulation of the same system. The temperature increase $\Delta \mathrm{T}(\mathrm{r}, \mathrm{z})$ is studied along horizontal cut lines at different height levels in the membrane. Our model perfectly fits simulation results in the whole homogeneous membrane excluding the heat generation zone which results from our hypothesis of a point heat source.

To emphasis the simplicity of this model, the truncated expression of $\xi\left(r^{*}, R^{*}, z^{*}\right)$ can been tabulated with respect to the normalized parameters. The temperature can be calculated only by using this abacus and without any numerical computing environment. This tabulated function is represented for various window radii on the Fig. 5. We have developed a simple model and a tabulated function that can predict the temperature in an X-ray transmission window system except in the heat generation zone. This zone where the electronic beam is impinging the target is however of great importance in the transmission X-ray system because it experiences the higher temperatures in the system. An approach to determine the heat distribution in this critical region is developed in the next section.

\subsection{Heat source modeling}

To extend this model in the heat generation zone, the point source is replaced by a half-sphere of radius $r_{s}$. The heat generated in the target is caused by the absorption of electrons in the interaction volume. As the $\mathrm{X}$-ray tubes considered have small focus spots, the heat source extension can be defined as the whole electron-matter interaction volume which is taken to be a portion of the sphere of interaction. Common acceleration energies in transmission tubes for medical/industrial application range between $30 \mathrm{keV}$ to $200 \mathrm{keV}$. For those energies and
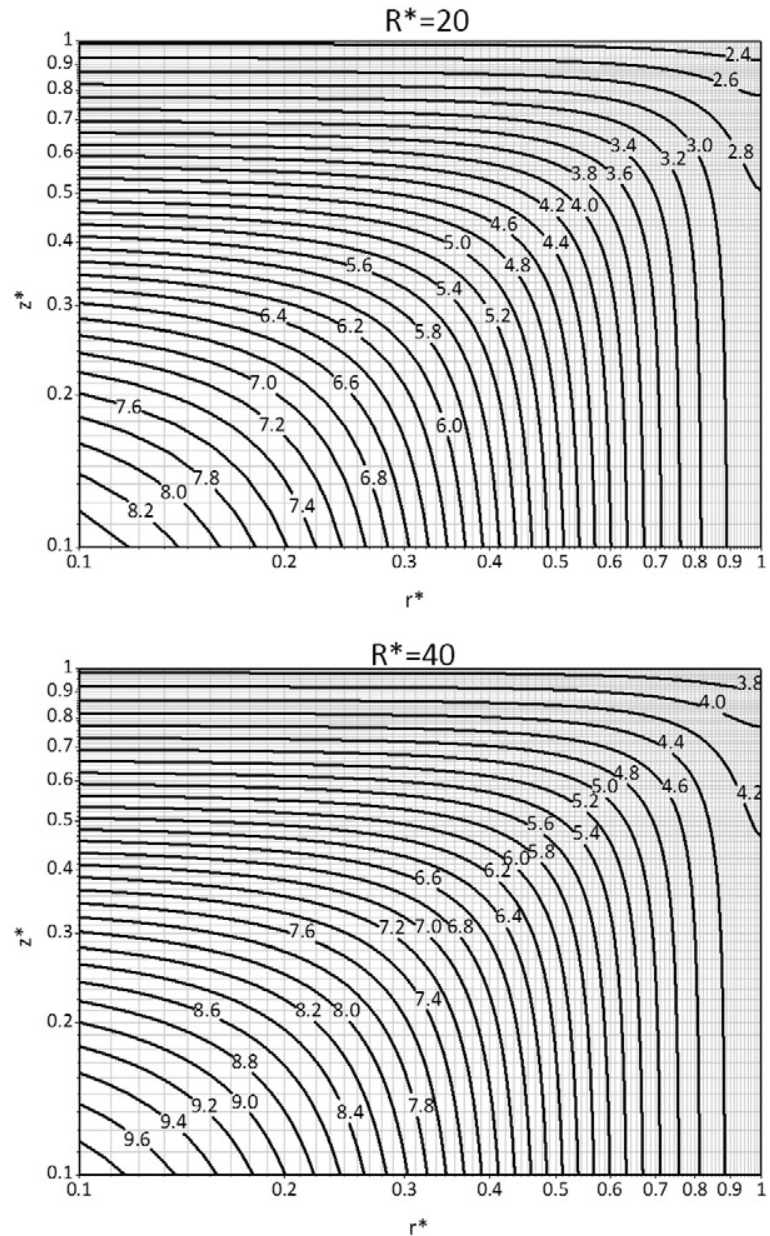

Fig. 5. Tabulation of the function $\xi$ for the parameters $\left(r^{*}, z^{*}\right)$ normalized with respect to the window height $\mathrm{H}$. The four plots refer to four different window aspect ratios R/H. 


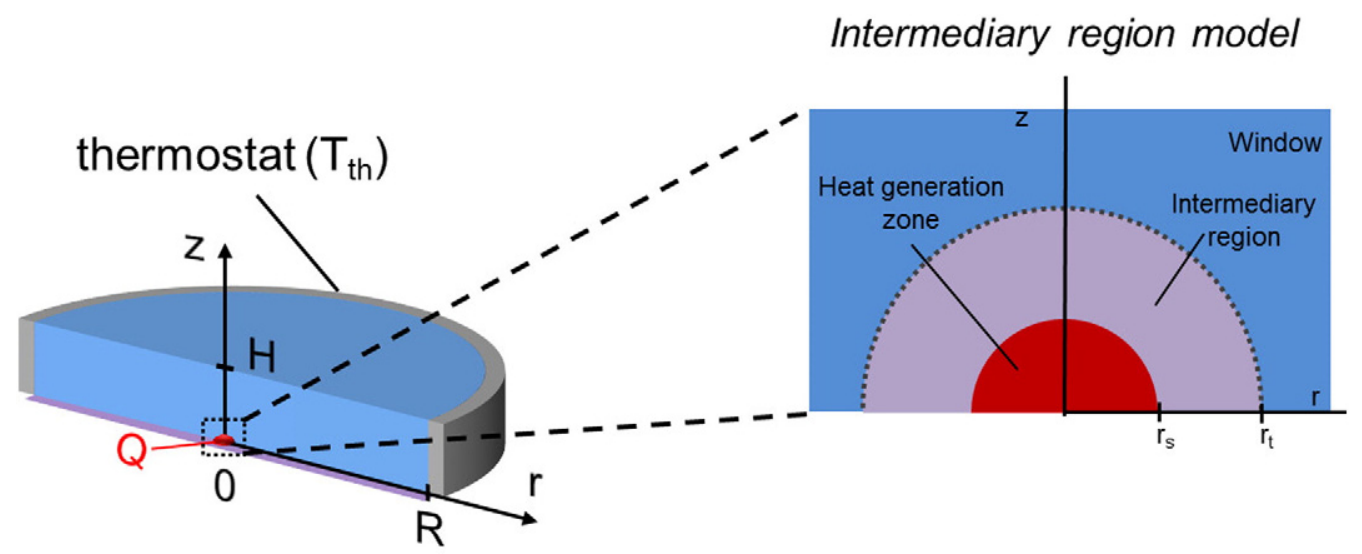

Target/window real system

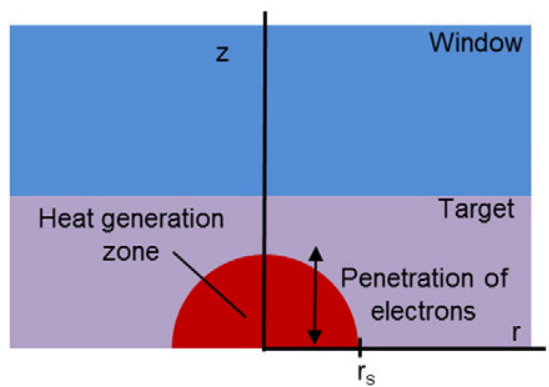

Fig. 6. Sketch of the geometry used to describe a real target/window system with conductivity $\lambda_{\mathrm{w}}$ and $\lambda_{\mathrm{t}}$.

considering heavy metals as required for our application, the depth of complete diffusion is small compared to the range of electrons. So the absorption volume can be considered hemispherical [10,11,15-17]. The range of the electrons is obviously energy-dependent and it directly determines the radius of the heat source. For tungsten, at $50 \mathrm{keV}$ the penetration depth of electrons is approximately $5 \mu \mathrm{m}[10,11]$. A sketch of this configuration is presented in Fig. 6 . To account for the presence of this volume distributed source in the model, the steady state Poisson's equation must be solved for a spherical system:

$\frac{d T^{2}}{d \rho^{2}}+\frac{2}{\rho} \frac{d T}{d \rho}=\mathrm{P}(\rho)$

where $\left(\rho=\sqrt{\mathrm{r}^{2}+\mathrm{z}^{2}}\right)$ is the spherical coordinate. The density power could be considered uniform but a simple way to account for different beam profiles is to write it as a polynomial function of the radial coordinate.

$\mathrm{P}(\rho)=\sum_{n=0}^{\infty} \alpha_{n} \rho^{n}$

Using Eqs. (7) and (8), the temperature distribution in the halfsphere of radius $r_{s}$ can be derived. Let's consider this region to have the target thermal conductivity $\lambda_{t}$. The window is assumed to present a thermal conductivity denoted $\lambda_{w}$. The thermal resistance of the window between a sphere of radius $r_{s}$ and the lateral thermostat must be added from expression 3 . Then the temperature in this spherical zone is:

$\Delta T(\rho)=\frac{\mathrm{Q} \cdot \xi\left(r_{s}^{*}, \mathrm{R}^{*}, 0\right)}{2 \pi \mathrm{H} \lambda_{w}}+\mathrm{Q} \sum_{n=0}^{\infty} \frac{\alpha_{n}\left(r_{s}{ }^{n+2}-\rho^{n+2}\right)}{(n+2)(n+3) \lambda_{t}}$.

The first term is the thermal resistance of the window and the second term is the contribution of the heat source region. Gaussian distribution is sometimes used to describe the electronic beam profile [10] and to model Gaussian as well as uniform heat generation; an even 8th degree polynomial function can be used. Table 1 presents the polynomial coefficients that fit several configurations. To account for a constant total power for both uniform and Gaussian beams, the $\alpha_{n}$ must be multiplied by a scaling factor presented on the right side of Table 1 . We can point out the simplest case of a uniform power density of: $P_{0}=\frac{3 Q}{2 \pi r_{1}{ }^{3}}$ which lead to the temperature $\Delta T_{\text {uniform }}$ from Eq. (9):

$\Delta T_{\text {uniform }}(\rho)=\frac{\mathrm{Q} \cdot \xi\left(r_{s}^{*}, \mathrm{R}^{*}, 0\right)}{2 \pi \mathrm{H} \lambda_{w}}+\frac{\mathrm{Q}}{4 \pi \lambda_{t} r_{s}}\left[1-\frac{\rho 2}{r_{s}^{2}}\right]$

Fig. 7 presents this temperature along the vertical axis of the heat generation zone for a uniform power density and Gaussian power density with different parameters. As expected, the maximum temperature

Table 1

Fitting parameters for the power density for two different kinds of beams: Gaussians and uniform. All the coefficients must be multiplied by the factor on the right.

\begin{tabular}{|c|c|c|c|c|c|c|}
\hline $\mathrm{n}$ & 8 & 6 & 4 & 2 & 1 & \\
\hline Coefficient & $\alpha_{8}\left[\mathrm{~m}^{-8}\right]$ & $\alpha_{6}\left[\mathrm{~m}^{-6}\right]$ & $\alpha_{4}\left[\mathrm{~m}^{-4}\right]$ & $\overline{\alpha_{2}\left[\mathrm{~m}^{-2}\right]}$ & $\overline{\alpha_{0}}$ & iviutipies \\
\hline Uniform beam & 0 & 0 & 0 & 0 & 1 & $\frac{3}{2 \pi r_{s}^{3}}$ \\
\hline $\begin{array}{l}\text { Gaussian beam } \\
\quad(\text { FWHM }=\sigma)\end{array}$ & $5.322 \cdot 10^{-4}$ & $-1.252 \cdot 10^{-2}$ & $1.129 \cdot 10^{-1}$ & -0.5 & 1 & $\frac{2}{(2 \pi)^{3 / 2} \sigma^{n+3}}$ \\
\hline
\end{tabular}




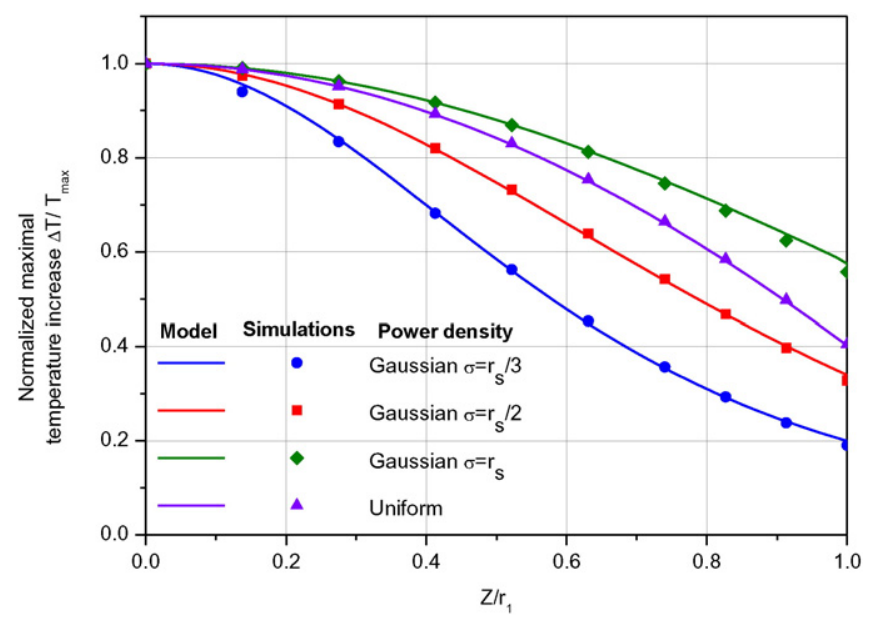

Fig. 7. Temperature increase along the cylinder axis (z-axis) within the heat generation zone of radius $r_{1}$. Tungsten target and diamond window of thickness $\mathrm{H}=100 \mu \mathrm{m}$ and radius $\mathrm{R}=4 \mathrm{~mm}$. The markers refer to finite element simulations while the solid lines refer to our model results.

in the heat generation zone occur at $(r, z)=(0,0)$ whereas it is not the case when the complete diffusion depth of electron is not small compared to the range (for higher energies for instance). This maximum temperature can be expressed from expression (10) as:

$\Delta T_{\max }=\frac{\mathrm{Q}}{2 \pi \mathrm{H}}\left(\frac{\xi\left(r_{s}^{*}, \mathrm{R}^{*}, 0\right)}{\lambda_{w}}+\frac{\mathrm{H}}{2 \lambda_{t} r_{s}}\right)$.

\subsection{Thermal resistance of the target}

Expression (11) is used to determine the temperature within the heat generation zone. Though, when using an X-ray tube at a given energy, the penetration depth is slightly inferior to the target thickness and some heat transfers occur in the target outside the heat generation zone (see Fig. 6). To account for this kind of conduction near the heat source a second hemispherical zone of radius $r_{t}$ and of conductivity $\lambda_{t}$ can be defined. This area in which radial conduction without heat generation occurs is represented in Fig. 6 and referred as intermediary zone. It represents the thermal resistance of the tungsten target and vanish for $r_{t}=r_{s}$. The maximum temperature can then be written as:

$\Delta T_{\max }=\frac{\mathrm{Q}}{2 \pi \mathrm{H}}\left(\frac{\xi\left(r_{t}{ }^{*}, \mathrm{R}^{*}, 0\right)}{\lambda_{w}}+\frac{\mathrm{H}}{\lambda_{t}}\left[\frac{3}{2 r_{s}}-\frac{1}{r_{t}}\right]\right)$.

The last term of Eq. (12) now corresponds to the temperature increase due to the thermal resistance of the bulk of the target. However, targets deposited on the window are cylindrical, not spherical and a particular attention is paid to adjust $r_{t}$ to match the geometry (see Fig. 6). When comparing FEM simulations of a cylindrical target to the model with a spherical target, the best agreement is found when $r_{t}$ is 1.5 times that of the cylindrical tungsten thickness. Fig. 8 presents the dependence of this maximal temperature increase on the geometrical ratio $\mathrm{R} / \mathrm{H}$ of the window with FEM simulations. This result is plotted for several target thicknesses ranging from 6 to $12 \mu \mathrm{m}$ and for a uniform power density. A comparison is made with FEM simulations performed on a realistic window/target system. After adjusting $r_{t}$ our model results fit perfectly with the simulations.

\subsection{Thermal boundary resistance}

The contact between the tungsten target and the diamond window is of great importance because of the phonon scattering that may occur at the interface [18]. Despite the atomically perfect interface

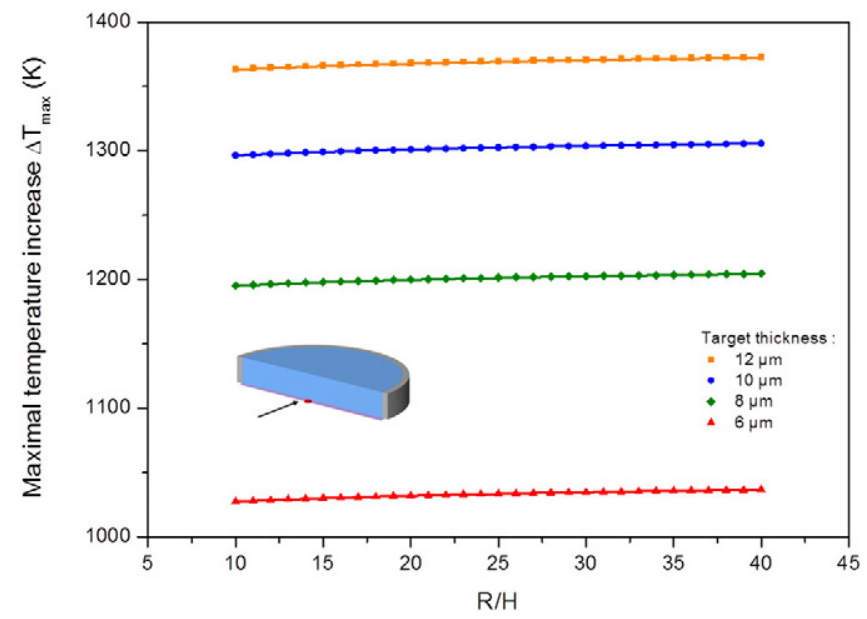

Fig. 8. Dependence of the maximal temperature increase in the window on the $\mathrm{R} / \mathrm{H}$ ratio. This dependence is plotted for several target thicknesses: $6 \mu \mathrm{m}$ (red), $8 \mu \mathrm{m}$ (green), $10 \mu \mathrm{m}$ (blue) and $12 \mu \mathrm{m}$ (yellow) and for both FE simulations (markers) and our model (solid lines)

considered here, the conduction through this surface does not correspond to a perfect contact. Taking a spherical target a radius $r_{t}$ and an interface conductance $G_{t b r}$, an additional term has been added into expression (13) to describe the maximum temperature increase of the system:

$\Delta T_{\max }=\frac{\mathrm{Q}}{2 \pi \mathrm{H}}\left(\frac{\xi\left(r_{t}{ }^{*}, \mathrm{R}^{*}, 0\right)}{\lambda_{w}}+\frac{\mathrm{H}}{\lambda_{t}}\left[\frac{3}{2 r_{s}}-\frac{1}{r_{t}}\right]+\frac{\mathrm{H}}{2 G_{t b r} r_{t}{ }^{2}}\right)$.

Large discrepancies are observed for experimental conductance measured at the tungsten-diamond interface and reported values scale from $40 \mathrm{MW} \cdot \mathrm{m}^{-2} \cdot \mathrm{K}^{-1}$ to $200 \mathrm{MW} \cdot \mathrm{m}^{-2} \cdot \mathrm{K}^{-1}[19,20]$. It has been shown that this conductance is very dependent on the surface properties (as roughness and crystalline orientation), on the deposition method and on the heat treatment applied [21-23]. In this model, a mean thermal boundary conductance of $100 \mathrm{MW} \cdot \mathrm{m}^{-2} \cdot \mathrm{K}^{-1}$ is assumed to represent the interface between a tungsten layer and a smooth single crystal diamond surface.

\subsection{Temperature dependence of the thermal conductivity}

Under operational conditions, local temperatures in the source can be increased by several hundreds of degrees. This model should take into account the thermal conductivity variations in the hottest part of the system, i.e. the heat source region. Under this assumption the heat equation becomes non-linear and a convenient approach to deal with this problem is to use the Kirchhoff's [24]. Let's note $\theta$ as the transformed temperature. Replacing in the heat equation results in a linear equation in $\theta$ that can be solved in the same previous way.

$\theta_{\max }=\frac{\mathrm{Q}}{2 \pi \mathrm{H}}\left(\frac{\xi\left(r_{t}^{*}, \mathrm{R}^{*}, 0\right)}{\lambda_{w}}+\frac{\mathrm{H}}{\lambda_{t}}\left[\frac{3}{2 r_{s}}-\frac{1}{r_{t}}\right]+\frac{\mathrm{H}}{G_{t b r} r_{t}^{2}}\right)$

The inverse transformation is then used to get the real temperature from $\theta$. At high temperature in diamond, the heat transfers are known to be limited by phonon-phonon interactions and the thermal conductivity is assumed to vary as $\lambda(T)=k T^{\alpha}$ with $\alpha$ being close to -1 cite $[25,26]$. $T$ being the local temperature expressed in Kelvins. In this model, we are only interested in single crystal diamond windows in which conductivity may slightly depend on chemical impurities and structural defect content but is around $2000 \mathrm{~W} \cdot \mathrm{m}^{-1} \cdot \mathrm{K}^{-1}$ at room temperature $[27,28]$. The experimental data at higher temperature have been extracted from experiments on IIa single crystal 


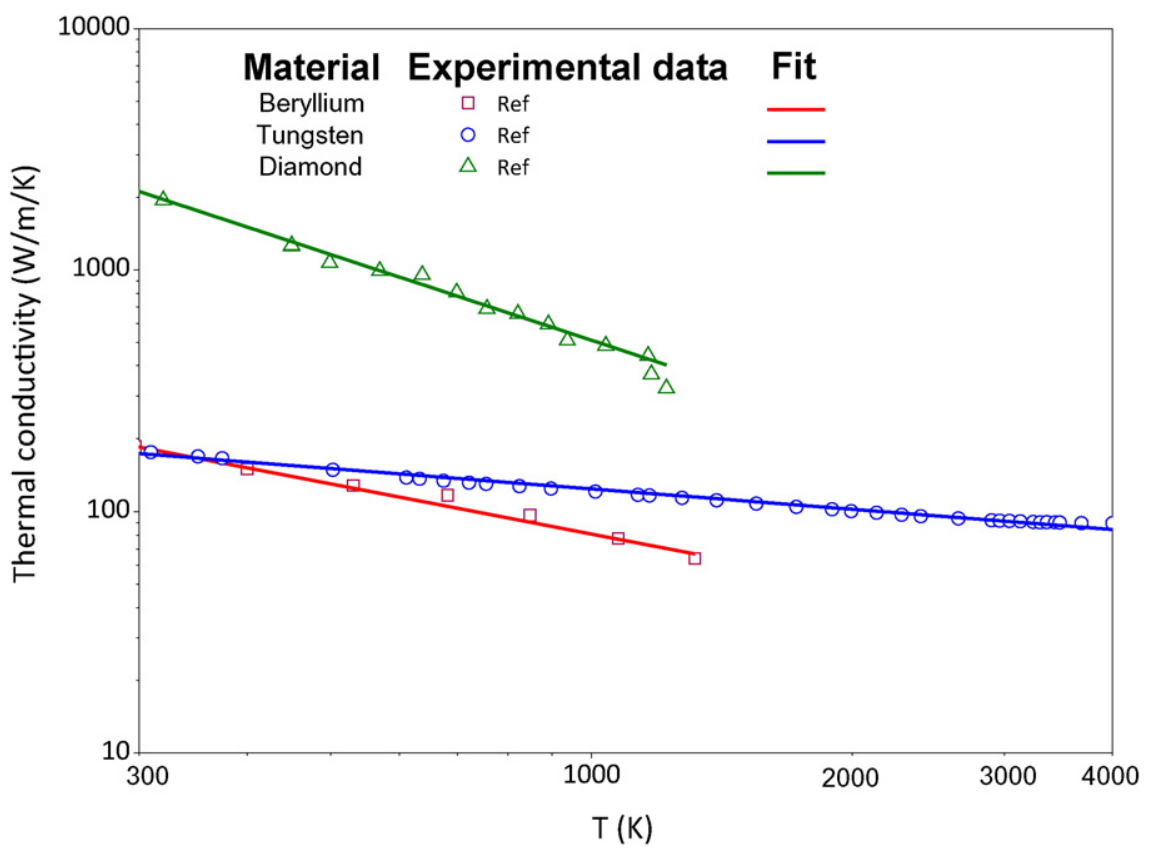

Fig. 9. Experimental data and fitted conductivities for beryllium, tungsten and polycrystalline diamond (in-plane) according to the references and parameters given in Table 2.

Table 2

Fitting parameters for the temperature dependence of the thermal conductivity of typical materials. Those parameters are calculated from experimental data extracted from the literature.

\begin{tabular}{lllll}
\hline Material & $\mathrm{k}$ & $\alpha$ & Ref. & Range \\
\hline Tungsten & 860 & -0.28 & {$[8]$} & {$[300 \mathrm{~K} ; 3800 \mathrm{~K}]$} \\
Diamond & $1.77 \cdot 10^{6}$ & -1.18 & {$[26,29,30]$} & {$[300 \mathrm{~K} ; 1000 \mathrm{~K}]$} \\
Beryllium & 9484 & -0.69 & {$[4]$} & {$[300 \mathrm{~K} ; 1200 \mathrm{~K}]$} \\
\hline
\end{tabular}

diamonds at different temperatures [29,26,30]. They represent an ideal model for the thermal conductivity of diamond windows between $300 \mathrm{~K}$ and $1200 \mathrm{~K}$. Table 2 shows different values of the fitting parameters for tungsten, beryllium and diamond Ila single crystal. The corresponding conductivities are plotted against the temperature in Fig. 9. For the particular form chosen for the thermal conductivity dependence, the maximal temperature increase of the system can be inversed from Eq. (14) (see details in Appendix C) and the maximal temperature is:

$\Delta T_{\max }=\left(\frac{\mathrm{Q} \lambda_{w, \text { th }}(\alpha+1)}{k}\left(\frac{\xi\left(r_{t}^{*}, \mathrm{R}^{*}, 0\right)}{2 \pi \mathrm{H} \lambda_{w, \mathrm{th}}}+\frac{1}{2 \pi \lambda_{t, \mathrm{th}}}\left[\frac{3}{2 r_{s}}-\frac{1}{r_{t}}+\frac{\lambda_{t, \mathrm{th}}}{G_{t b r} r_{t}^{2}}\right]\right)+T_{t h}{ }^{\alpha+1}\right)^{1 /(\alpha+1)}-T_{\text {th }}$

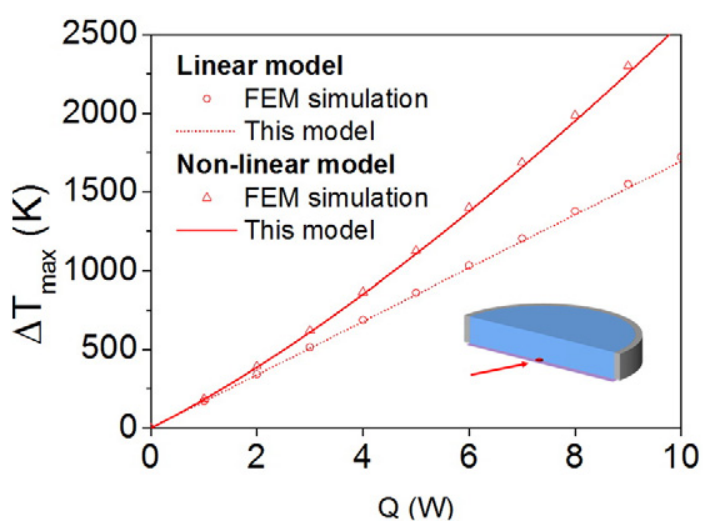

where $\lambda_{w, t h}$ and $\lambda_{t, t h}$ are the thermal conductivities of the window and target material at room temperature and $\alpha$ and $k$ are the fitting parameter of the target conductivity. $\mathrm{Q}$ is the source power, $\mathrm{H}$ and $\mathrm{R}$ are respectively the window height and radius. $r_{s}$ refers to the heat source radius and $r_{t}$ to the intermediary region described at the end of Section 2.2. $T_{t h}$ is the thermostat temperature at the edge of the window. $G_{t b r}$ is the thermal boundary conductance of the target/window interface and is considered constant over the temperature range. In fact, this parameter increases with temperature which means that the worst case scenario is modeled by taking it constant [31]. Using Eq. (14) and the previous FEM simulation a comparison of the maximal temperature is possible as a function of the generated heat power. A $100 \mu \mathrm{m}$ thick diamond window of $8 \mathrm{~mm}$ diameter with a $6 \mu \mathrm{m}$ thick target made of tungsten is modeled. Fig. 10 shows the correlation for both constant (Eq. (13), $\lambda_{t}=2113 \mathrm{~W} \cdot \mathrm{m}^{-1} \cdot \mathrm{K}^{-1}$ ) and non-linear (Eq. (15), $\lambda_{t}=\mathrm{kT}^{\alpha}$ ) models with simulations that use experimental data points for the definition of conductivity. As soon as the temperature overcomes the thermostat temperature by $200 \mathrm{~K}$ the non-linear model exhibits a relatively different behavior leading in potential misinterpretations when using a linear model.

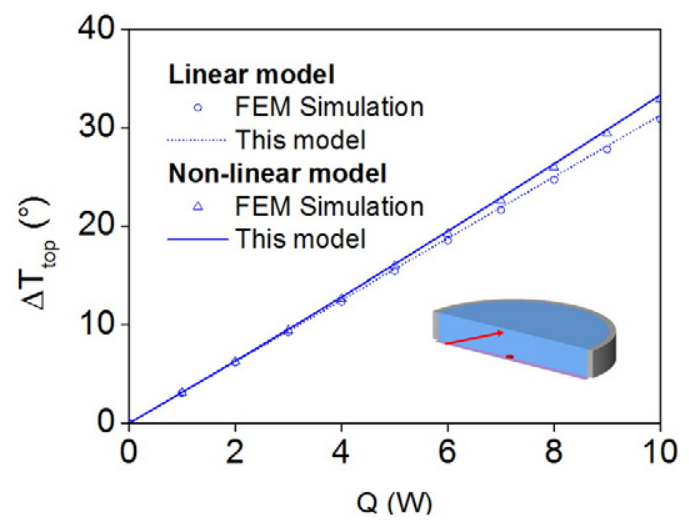

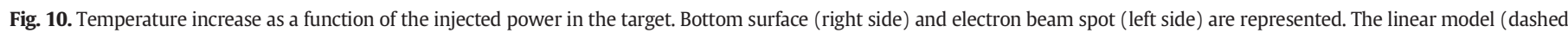
line) includes a constant $\lambda$ and the no-linear-model (solid line) includes the temperature dependence. The results of FEM simulations are also represented (markers). 


\section{Discussion}

Calculating the analytical temperature distribution for systems with axial symmetry is often achieved by solving the steady state heat equation in cylindrical coordinates assuming a solution with separated variables [9,32]. The resulting expression involves Bessel-Fourier series whose coefficients are calculated with the boundary conditions of the system. Those solutions suffer from poor or restricting definitions and cannot always be approximated. Such problems have also been addressed by using direct and inverse Hankel transformations also involving Bessel's functions [15,33], but in most cases the heat source is only defined on the target surface excluding any volume power dissipation. Defining the heat source within the target volume is a more realistic approach for our system.

The top side of the window is generally subjected to atmospheric pressure (e.g. to a free or forced air convection). When it is neglected, this side of the window shows temperatures near the ambient as presented in Section 2.1. Thus almost no contribution of convective transfers to the overall heat transfer is expected. Additionally, the radiative effect is of particular importance on the surfaces at elevated temperatures. But even when radiative transfers are neglected, the surfaces that are radiating a non-negligible flux are considerably small compared to the conductive ones and the contribution of this phenomenon should be very small. To check that both radiative and convective transfers can be neglected in this model without too much error, the system has been modeled using FEM simulations and the heat flux magnitudes have been simulated on the top and bottom sides of the window. As presented in Fig. 11 the radiative flux as well as the convective flux stand several orders of magnitude below the conductive flux and can therefore be ignored in the whole model. Indeed, no changes can be seen from the sole conduction model (results not shown here) when adding free convection with air, forced convection with air or oil (fluid velocity of $10 \mathrm{~m} / \mathrm{s}$ ) or radiation.

At very high power densities, if the temperature rises above the fusion temperature of the anode a spherical fused zone can been noticed on the target surface. The variation of the size of this fused zone has been studied for thick reflection target of tungsten, molybdenum and copper with respect to the power of the impinging electron beam [12].

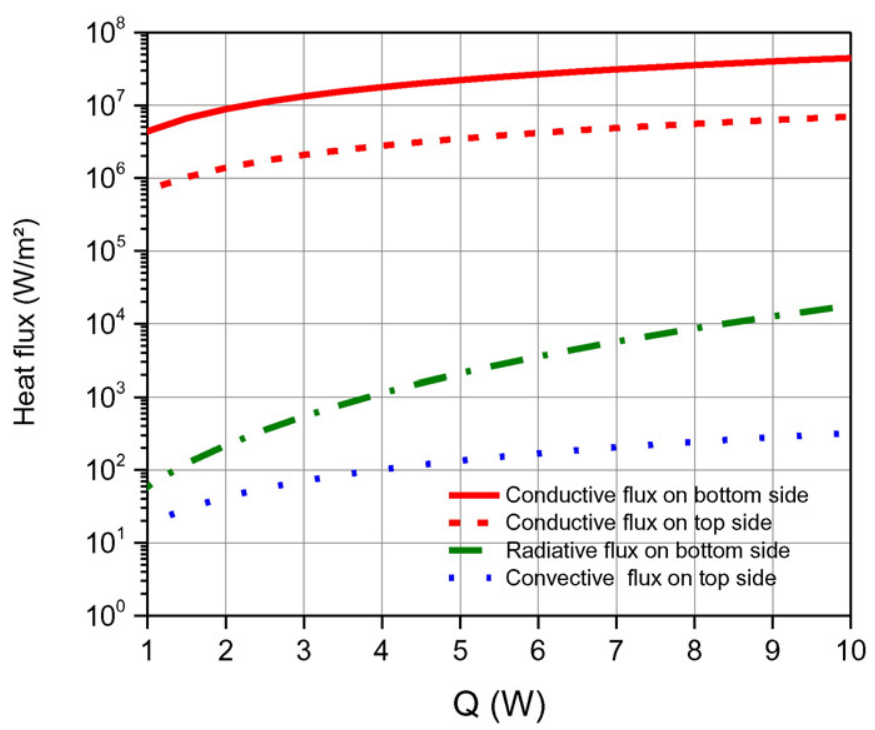

Fig. 11. Heat flux magnitude calculated from simulations and plotted as a function of the power of the tube. The radiative flux is calculated from the bottom side of the window $(z=0)$, the convective flux is calculated on the top side with an external free convection of air. The conductive flux is calculated on both surfaces (red solid and dashed lines) and also plotted against the power of the tube. The window is $8 \mathrm{~mm}$ in diameter and $100 \mu \mathrm{m}$ thick

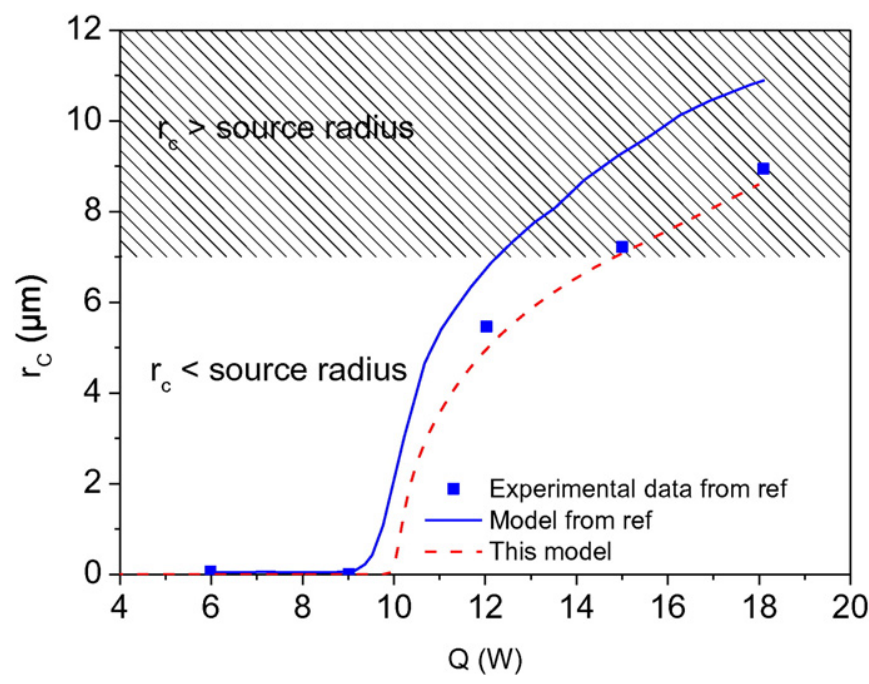

Fig. 12. Our model (Eq. (7)) for $r_{c}$ (dashed line) plotted along with experimental data measured by Grider et al. [12]. The system studied is a plain tungsten target with a $7 \mu \mathrm{m}$ radius impinging electron beam. The shaded area corresponds to a critical radius larger than the heat generation zone (see details in the annexes). The solid blue line represents an analytical model for reflection targets exposed ref. [12].

There are actually two potential regimes depending on where the melting process occurs. Assuming that this fusion process takes place in the heat generation zone (the hottest region of the system) one can determine the radius of this fused zone as a function of the input power of the tube (see details in Appendix D).

$\mathrm{r}_{\mathrm{c}}=\sqrt{6 \lambda_{t} \theta_{f}\left(\frac{1}{\mathrm{P}_{\max }}-\frac{1}{\mathrm{P}}\right)}$

where $r_{c}$ represents the critical melting radius, $P$ is the power density injected and $\mathrm{P}_{\max }$ is the maximum power density allowable before fusion of the target. If this fusion process extends out of the heat generation zone (in the intermediary region), the previous expression is calculated with a different expression (see details in Appendix D). Fig. 12 shows the correlation between the critical melting radius obtained from Eq. (15), data from another existing model and the experimental data from reference [12] for a plain tungsten target (reflection target) of $1 \mathrm{~cm}$ diameter and $2.5 \mathrm{~cm}$ height for a given source radius $r_{s}$ of $7 \mu \mathrm{m}$.

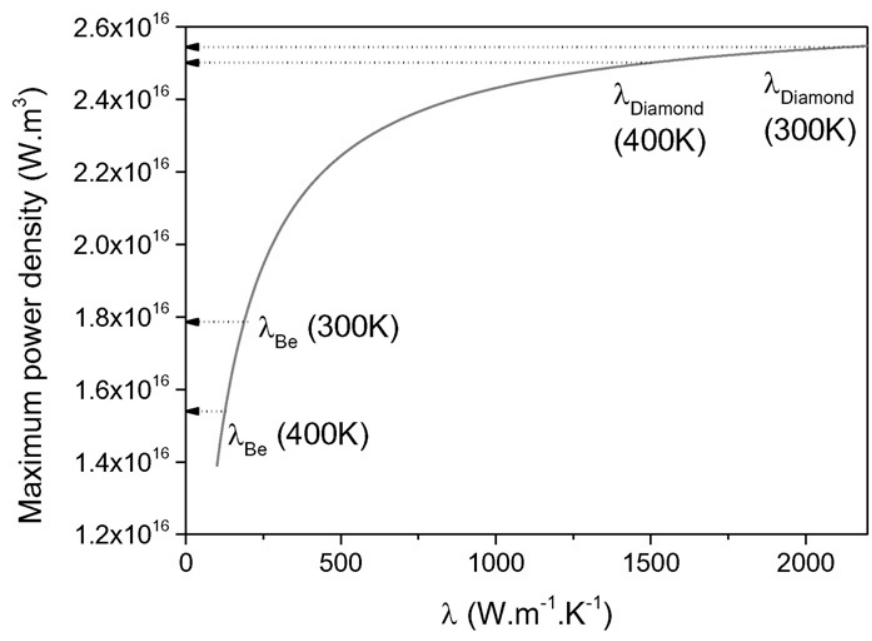

Fig. 13. Maximal allowable power density in the tube as a function of the thermal conductivity of the window. The window is $8 \mathrm{~mm}$ in diameter and $100 \mu \mathrm{m}$ in height and the source radius is $6 \mu \mathrm{m}$. 


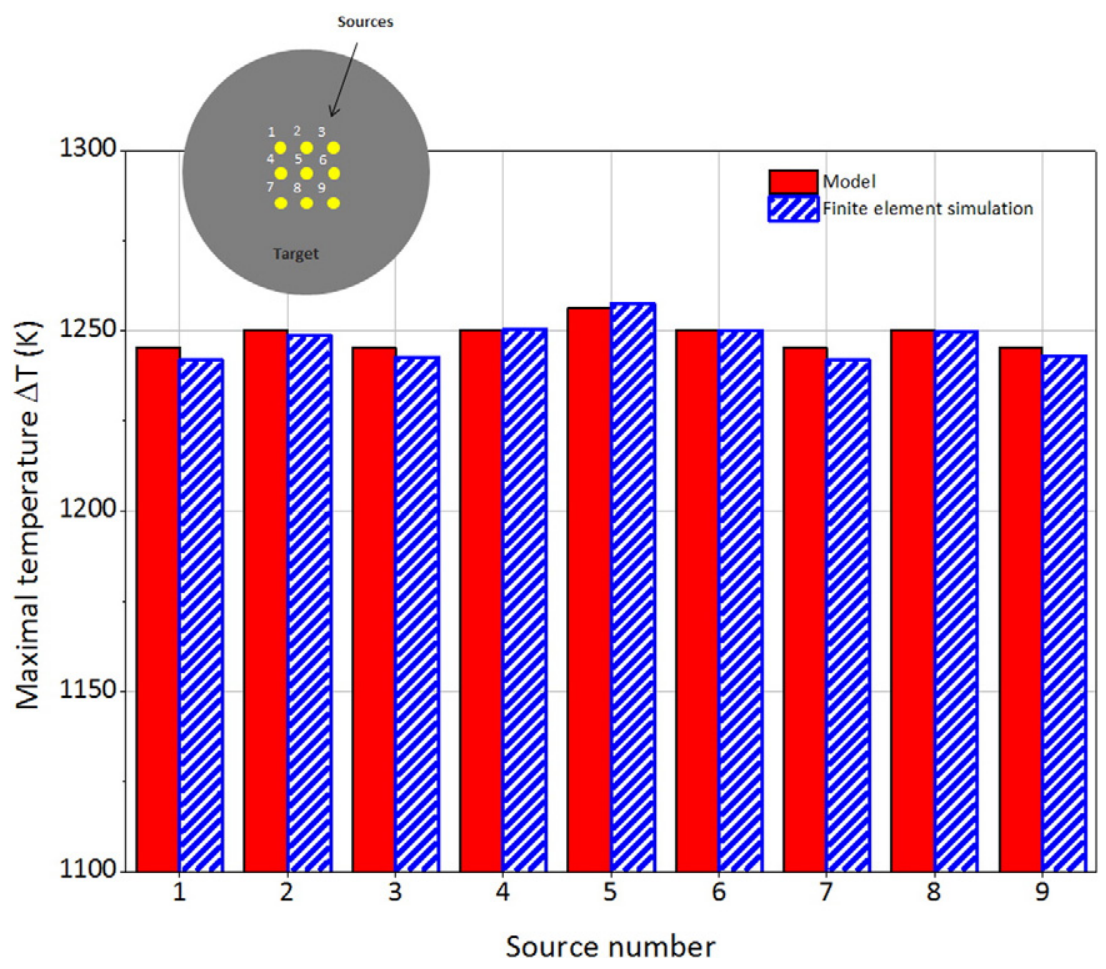

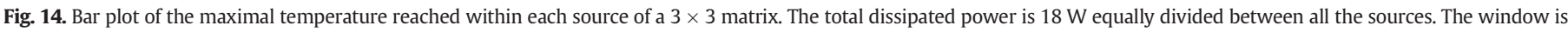
$100 \mu \mathrm{m}$ thick with a $10 \mu \mathrm{m}$ tungsten target and each source is $3.5 \mu \mathrm{m}$ in diameter and spaced from its nearest neighbor by $50 \mu \mathrm{m}$.

For powers below a certain value, there is no critical radius since the target fusion temperature is not reached. From expression (7), one can know the maximal power density allowable in a system without occurrence of a melting process (i.e. $r_{c}=0$ ). The power density from which a critical radius $r_{c}>0$ is referred as $P_{\max }$. Fig. 13 shows a plot $P_{\max }$ as a function of the thermal conductivity of the window. The $\mathrm{P}_{\max }$ value for diamond is higher than the beryllium one, indicating the high thermal spreading efficiency of diamond windows and the possibility to operate an X-ray tube at a higher current/voltage with a diamond window. Because of the choice of the fitting parameter for diamond, the results presented here only apply to single crystal diamond windows. In the case of polycrystalline diamond, the thermal conductivity is anisotropic and highly dependent on the grain distribution [6,7,34,25]. Anisotropy of $50-60 \%$ can be observed between normal and tangential conductivity with tangential conductivity close to $2000 \mathrm{~W} \cdot \mathrm{m}^{-1} \cdot \mathrm{K}^{-1}$ at room temperature for good quality diamond. The large discrepancies observed in the literature for different quality and thickness of polycrystalline diamond films prevent a simple use of this model on this material. When looking at the thermal conductivity of beryllium and single crystal diamond at a typical working temperature of $400 \mathrm{~K}$ in Fig. 12, the

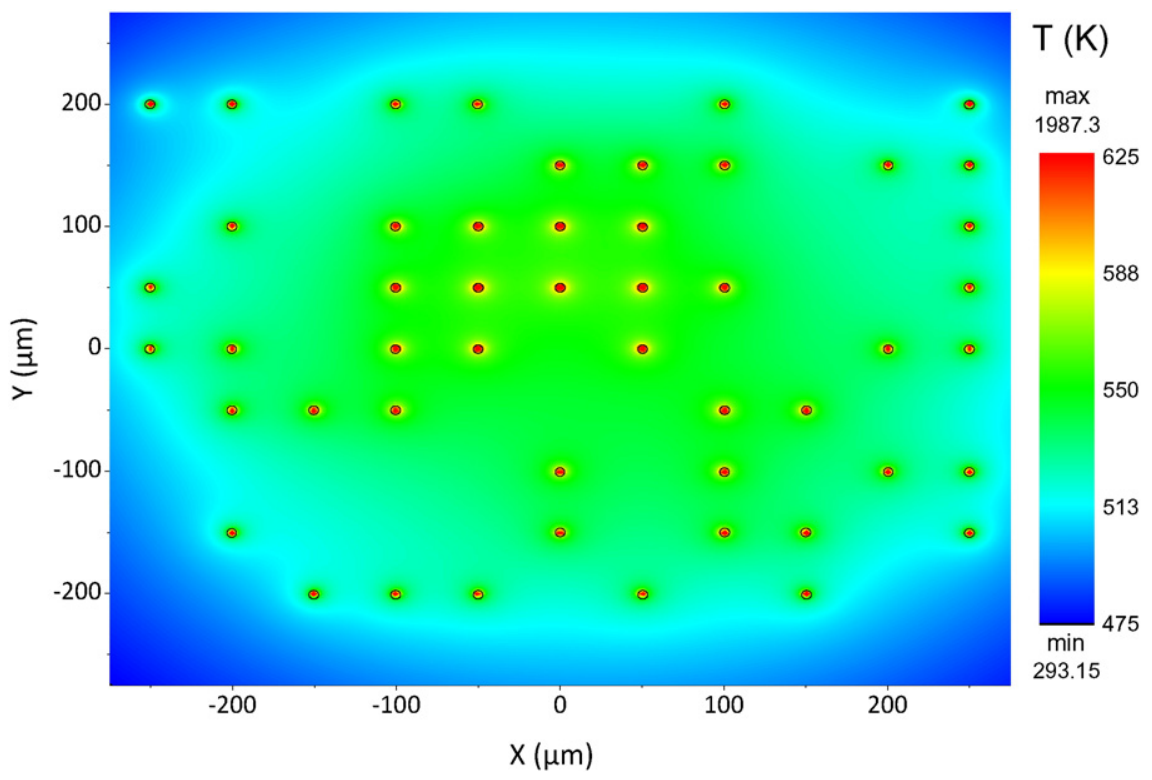

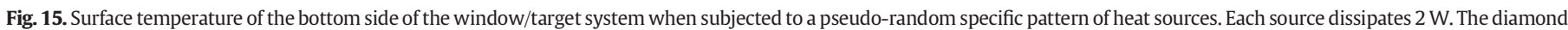
window is $100 \mu \mathrm{m}$ thick supporting a $10 \mu \mathrm{m}$ tungsten target and each source is $3.5 \mu \mathrm{m}$ in diameter and spaced from its nearest neighbor by $50 \mu \mathrm{m}$. 
diamond window allows a maximal power density 1.62 times superior as the one allowed by a beryllium window.

\section{Application to multisource $\mathrm{X}$-ray systems}

Recent developments have shown that imaging with an array of distributed X-ray sources is a promising alternative to standard techniques as it can give extremely good resolution and multiplexing capabilities. In such applications a patterned X-ray generation with small size sources is used resulting in a similar patterned heat generation. By using a linear superposition of the solution for several sources, distributed in a specific pattern, the overall temperature distribution is easily calculated. For matters of comparison, a $3 \times 3$ matrix of $3.5 \mu$ m heat sources spaced by $50 \mu \mathrm{m}$ has been modeled and the maximal temperature within each source is calculated in the model and the FEM simulation. Fig. 14 shows the good similarity of our calculations. The main advantage of such a simple model is to use it on more complex problems with a substantial gain on the computing time and resources. An example can be seen in Fig. 15 where a complex pseudo-random matrix of sources is modeled and the surface temperature on the bottom side of the target is displayed. It is an application of coded aperture X-ray tube in which the patterned target acts as an irregular array of heat sources. Fig. 15 is obtained in few minutes with a standard computer while the complete FEM simulation of this system would take more time and resources.

\section{Conclusion}

A novel approach has been used to derive a thermal model able to describe the temperature at high density power in diamond X-ray transmission windows. It could be used with several heat sources to model multi-target X-ray tubes or more generally in lateral heat spreaders with distributed small sized source systems. Among the different applications aimed for this model, diamond windows for nanofocus X-ray tubes are of great importance. This new model has been compared to finite element simulations and to experimental data from the literature. Undergoing developments on monocrystalline diamond windows will eventually lead to experimental comparison. It provides flexible and fast evaluation of the temperature with a controlled physical accuracy to bring a simpler vision to the field of thermal management in X-ray tubes.

\section{Prime novelty statement}

In this paper, an analytical model has been developed to complement finite element simulations and to bring a simpler but accurate vision to the field of thermal management in X-ray tubes. This analytical model allows readily modeling of more and more challenging systems by significantly reducing the computing time, thus enabling wider parameter range evaluation for engineering phase. Moreover, there are currently no simple analytical models able to describe the thermal behavior of diamond windows in small focus X-ray tubes working in transmission configuration.

\section{Appendix A. Study of the convergence of the series}

In order to prove the convergence of the series defined in the first section of this article one has to use an integral test for convergence. Let's write the series we want to study:

$\sum_{-\infty}^{+\infty} U_{n}=\sum_{-\infty}^{+\infty} f(n)$

where the function $\mathrm{f}$ is

$$
\begin{aligned}
& f: \mathbb{R} \rightarrow \mathbb{R} \\
& t \mapsto=\frac{1}{\sqrt{\frac{\mathrm{r}^{2}}{\mathrm{H}^{2}}+\left(2 t-\frac{\mathrm{z}}{\mathrm{H}}\right)^{2}}}-\frac{1}{\sqrt{\frac{\mathrm{R}^{2}}{\mathrm{H}^{2}}+\left(2 t-\frac{\mathrm{z}}{\mathrm{H}}\right)^{2}}} .
\end{aligned}
$$

This double series can also be written as two distinct series:

$$
\sum_{-\infty}^{+\infty} f(n)=\sum_{0}^{+\infty} f(n)+\sum_{0}^{+\infty} f(-n)-f(0) .
$$

Considering the first member of this partition and noticing that $U_{n}=f(n)$ and that $f(t)$ is a monotone decreasing function on the following interval $[0,+\infty$ [, an upper and a lower bound can be found for the general term of the series.

$\int_{n}^{n+1} f(t) d t<U_{n}<\int_{n-1}^{n} f(t) d t$.

By adding the terms of the series $N$ times, we obtain:

$\int_{0}^{N+1} f(t) d t<\sum_{n=0}^{N} U_{n}<U_{0}+\int_{0}^{N} f(t) d t$.

An antiderivative of the irrational function $f$ has to be found. We can rewrite

$f(t)=\frac{1}{\sqrt{a t^{2}+b t+c}}-\frac{1}{\sqrt{a t^{2}+b t+c^{\prime}}}$

with $a=4, b=\frac{4 \mathrm{z}}{\mathrm{H}}, c=\frac{\left(\mathrm{r}^{2}+\mathrm{z} 2\right)}{\mathrm{H}^{2}}$ and $c^{\prime}=\frac{\left(\mathrm{R}^{2}+\mathrm{z}^{2}\right)}{\mathrm{H}^{2}}$.

As $b^{2}-4 a c<0$ and for $r \neq 0$, we know that $\frac{1}{\sqrt{a}} \operatorname{argsh}\left(\frac{2 a t+b}{\sqrt{4 a c-b^{2}}}\right)-$ $\frac{1}{\sqrt{a}} \operatorname{argsh}\left(\frac{2 a t+b}{\sqrt{4 a c^{\prime}-b^{2}}}\right)$ is an antiderivative of this function.

The upper and lower bounds are now:

$A_{N}<\sum_{n=0}^{N} U_{n}<U_{0}+B_{N}$

where

$$
\begin{aligned}
A_{N}= & \frac{1}{2} \operatorname{argsh}\left(\frac{2(N+1) \mathrm{H}}{\mathrm{r}}+\frac{\mathrm{z}}{\mathrm{r}}\right)-\frac{1}{2} \operatorname{argsh}\left(\frac{2(N+1) \mathrm{H}}{\mathrm{R}}+\frac{\mathrm{z}}{\mathrm{R}}\right) \\
& +\frac{1}{2} \operatorname{argsh}\left(\frac{\mathrm{z}}{\mathrm{R}}\right)-\frac{1}{2} \operatorname{argsh}\left(\frac{\mathrm{z}}{\mathrm{r}}\right)
\end{aligned}
$$

$$
\begin{aligned}
B_{N}= & \frac{1}{2} \operatorname{argsh}\left(\frac{2 N H}{r}+\frac{z}{r}\right)-\frac{1}{2} \operatorname{argsh}\left(\frac{2 N H}{\mathrm{R}}+\frac{\mathrm{z}}{\mathrm{R}}\right) \\
& +\frac{1}{2} \operatorname{argsh}\left(\frac{\mathrm{z}}{\mathrm{R}}\right)-\frac{1}{2} \operatorname{argsh}\left(\frac{\mathrm{z}}{\mathrm{r}}\right) .
\end{aligned}
$$

For the sake of simplicity, we make use of the transformation argsh $(x)=\log \left(x+\sqrt{x^{2}-1}\right)$. When $N$ tends to infinity we can notice that 
$\lim _{N \rightarrow \infty}\left(A_{N}\right)=\lim _{N \rightarrow \infty}\left(A_{N}\right)=\frac{1}{2} \log \left[\frac{\left(\frac{\mathrm{z}}{\mathrm{r}}+\sqrt{\left(\frac{\mathrm{z}}{\mathrm{r}}\right)^{2}+\left(\frac{\mathrm{R}}{\mathrm{r}}\right)^{2}}\right)}{\left(\frac{\mathrm{z}}{\mathrm{r}}+\sqrt{\left(\frac{\mathrm{z}}{\mathrm{r}}\right)^{2}+1}\right)}\right]$.

Upper and lower bounds of the second series can be found in an analog manner and consequently the whole series have the following upper and lower bounds:

$\log \left[\frac{\mathrm{R}}{\mathrm{r}}\right]<\sum_{n=-\infty}^{+\infty} U_{n}<U_{0}+\log \left[\frac{\mathrm{R}}{\mathrm{r}}\right]$.

As the two bounds have finite limits when $N$ tends to infinity, the series studied is convergent and has a finite limit referred as $\xi$. The same method is applicable to the specific case of $r=0$ where the antiderivative for $f$ is slightly different.

$\xi=\lim _{n \rightarrow \infty} \sum_{n=-\infty}^{+\infty} U_{n}$

One can also notice that when the heat sources are far from the observation point, the temperature has a simple analytical form that match exactly the one described by the infinite 1D linear source problem.

$\lim _{\mathrm{r} \rightarrow \mathrm{R}} U_{0}=0$ and so $\xi=\log \left[\frac{\mathrm{R}}{\mathrm{r}}\right]$

\section{Appendix B. Truncating the series and evaluating the error}

As a consequence of the decreasing function $\mathrm{f}$ studied previously, the Pth terms of the series can be neglected as soon as P $\gg$ R. Let's study the series of the rest at the Pth order using another integral test.

$\sum_{\mathrm{P}=1}^{+\infty} r_{P}=\sum_{-\infty}^{+\infty} f(n)-\sum_{0}^{\mathrm{P}} f(n)+\sum_{1}^{\mathrm{P}} f(-n) \quad$ with $\mathrm{P} \gg \mathrm{R}$.

And if we note $R_{p}$ the limit of this convergent series

$R_{P}=\lim _{M \rightarrow \infty} \sum_{\mathrm{P}+1}^{M} r_{P}$.

Using the same integral test, one can write

$\sum_{\mathrm{P}+1}^{M} r_{p}<\int_{\mathrm{P}}^{M} f(t) d t+\int_{\mathrm{P}}^{M} f(-t) d t$.

And consequently, if $r \neq 0$

$R_{P}<\frac{1}{2} \log \left[\frac{\left(\frac{2 \mathrm{PH}}{\mathrm{r}}+\frac{\mathrm{z}}{\mathrm{r}}+\sqrt{\left(\frac{2 \mathrm{PH}}{\mathrm{r}}+\frac{\mathrm{z}}{\mathrm{r}}\right)^{2}+\left(\frac{\mathrm{R}}{\mathrm{r}}\right)^{2}}\right)}{\left(\frac{2 \mathrm{PH}}{\mathrm{r}}+\frac{\mathrm{z}}{\mathrm{r}}+\sqrt{\left(\frac{2 \mathrm{PH}}{\mathrm{r}}+\frac{\mathrm{z}}{\mathrm{r}}\right)^{2}+1}\right)} \cdot \frac{\left(\frac{2 \mathrm{PH}}{\mathrm{r}}-\frac{\mathrm{z}}{\mathrm{r}}+\sqrt{\left(\frac{2 \mathrm{PH}}{\mathrm{r}}-\frac{\mathrm{z}}{\mathrm{r}}\right)^{2}+\left(\frac{\mathrm{R}}{\mathrm{r}}\right)^{2}}\right)}{\left(\frac{2 \mathrm{PH}}{\mathrm{r}}-\frac{\mathrm{z}}{\mathrm{r}}+\sqrt{\left(\frac{2 \mathrm{PH}}{\mathrm{r}}-\frac{\mathrm{z}}{\mathrm{r}}\right)^{2}+1}\right)}\right]$.

This upper bound of the series can be used (except in $r=0$ where the upper bound has a different form) to determine the error made on the temperature when using P source instead of infinity of source. As expected this upper bound is decreasing to 0 when $P$ tends to infinity.

\section{Appendix C. Kirchhoff's transformation}

To solve the non-linear heat equation, the Kirchhoff's transformation of the temperature was used. The transformed temperature is defined as:

$\mathcal{T}(T)=\theta=\frac{1}{\lambda_{\text {th }}} \int_{\text {th }}^{T} \lambda\left(T^{\prime}\right) d T^{\prime}$

with $\lambda\left(T^{\prime}\right)=k T^{\alpha}$

As the conductivity dependence on $T$ is defined through a power law, the direct transformation writes:

$\theta=\frac{k}{\lambda_{t h}(\alpha+1)}\left(T^{\alpha+1}-T_{t h}{ }^{\alpha+1}\right)$

where $\lambda_{t h}$ refers to the room temperature thermal conductivity. Calculating $\frac{\partial \theta}{\partial r}$ et $\frac{\partial 2 \theta}{\partial r 2}$ we can notice that the second order non-linear equation in $T$ transforms into a linear equation in $\theta$. This equation is solved in the same previous way as for linear systems. Once the form of $\theta$ is known, the final expression of $T$ is found by solving the inverse transformation:

$T(\mathrm{r}, \mathrm{z})=\mathcal{T}^{-1}(\theta)=\left(\frac{\lambda_{t h}(\alpha+1) \theta_{\max }}{k}+T_{\text {th }}\right)^{1 /{ }_{(\alpha+1)}}$

This expression is the inverse Kirchhoff's transformation corresponding to $\theta$.

\section{Appendix D. Critical radius}

In the heat generation zone, at $\mathrm{z}=0$, the temperature increase writes:

$\Delta T(\mathrm{r}, \mathrm{z})=\frac{\mathrm{Q} \cdot \xi\left(\mathrm{r}_{\mathrm{t}}, \mathrm{R}, \mathrm{z}\right)}{2 \pi \mathrm{H} \lambda_{\mathrm{w}}}+\frac{\mathrm{Q}}{4 \pi \lambda_{t} r_{s}{ }^{3}}\left[r_{s}{ }^{2}-\mathrm{r}^{2}\right]+\frac{\mathrm{Q}}{2 \pi \lambda_{t}}\left[\frac{1}{r_{s}}-\frac{1}{r_{t}}+\frac{\lambda_{t}}{G_{t b r} r_{t}{ }^{2}}\right]$

Outside the heat generation zone but still in the target and close to the source, it is written:

$\Delta T(\mathrm{r}, \mathrm{z})=\frac{\mathrm{Q} \cdot \xi\left(\mathrm{r}_{\mathrm{t}}, \mathrm{R}, \mathrm{z}\right)}{2 \pi \mathrm{H} \lambda_{w}}+\frac{\mathrm{Q}}{2 \pi \lambda_{t}}\left(\frac{1}{\mathrm{r}}-\frac{1}{r_{t}}+\frac{\lambda_{t}}{G_{t b r} r_{t}^{2}}\right)$

The radius from which the temperature rises above the target fusion temperature is obtained by replacing $T(\mathrm{r}, \mathrm{z})$ by $T_{\text {fusion }}$ in both expressions. The limiting radial parameter $r$ is denoted $r_{c}$. Meaning that fusion of the target occurs for $r<r_{c}$. One can now express this critical value in the heat generation zone:

$\mathrm{r}_{\mathrm{c}}=\left(r_{s}{ }^{2}+4 \pi r_{s}{ }^{3} \lambda_{t}\left[\frac{\xi\left(r_{t}, \mathrm{R}, \mathrm{z}\right)}{2 \pi \mathrm{H} \lambda_{w}}+\frac{1}{2 \pi \lambda_{t}}\left[\frac{1}{r_{s}}-\frac{1}{r_{t}}+\frac{\lambda_{t}}{G_{t b r} r_{t}{ }^{2}}\right]-\theta_{f} / \mathrm{Q}\right]\right)^{1 / 2}$

By rearranging the factor a simpler form can be obtained:

$\mathrm{r}_{\mathrm{c}}=\sqrt{6 \lambda_{t} \theta_{f}\left(\frac{1}{\mathrm{P}_{\max }}-\frac{1}{\mathrm{P}}\right)}$ 
With

$$
\mathrm{P}_{\max }=\frac{6 \lambda_{t} \theta_{f}}{r_{s}{ }^{2}+4 \pi r_{s}{ }^{3} \lambda_{t}\left(\frac{\xi\left(r_{t}, \mathrm{R}, \mathrm{z}\right)}{2 \pi \mathrm{H} \lambda_{w}}+\frac{1}{2 \pi \lambda_{t}}\left[\frac{1}{r_{s}}-\frac{1}{r_{t}}+\frac{\lambda_{t}}{G_{t b r} r_{t}^{2}}\right]\right)} .
$$

And just outside this zone:

$r_{c}=\left(\frac{1}{r_{t}}-\frac{\lambda_{t}}{G_{t b r} r_{t}^{2}}-\frac{\lambda_{t} \xi\left(r_{t}, \mathrm{R}, \mathrm{z}\right)}{\mathrm{H} \lambda_{w}}+\frac{2 \pi \lambda_{t} \theta_{f}}{\mathrm{Q}}\right)^{-1}$

$\theta_{f}$ is the transformed temperature of the target melting point This parameter has been plot against the total power dissipated Q in Fig. 11. Finally, it is rewritten.

$\mathrm{r}_{\mathrm{c}}=\left\{\begin{array}{c}\sqrt{6 \lambda_{t} \theta_{f}\left(\frac{1}{\mathrm{P}_{\max }}-\frac{1}{\mathrm{P}}\right)}, \mathrm{r}_{\mathrm{c}} \leq r_{t} \\ \left(\frac{1}{r_{t}}-\frac{\lambda_{t}}{G_{t b r} r_{t}^{2}}-\frac{\lambda_{t} \xi\left(r_{t}, \mathrm{R}, \mathrm{z}\right)}{\mathrm{H} \lambda_{w}}+\frac{2 \pi \lambda_{t} \theta_{f}}{\mathrm{Q}}\right)^{-1}, \mathrm{r}_{\mathrm{c}}>r_{t}\end{array}\right\}$.

\section{References}

[1] O. Brunke, K. Brockdorf, S. Drews, B. Müller, T. Donath, J. Herzen, F. Beckmann, Comparison between X-ray tube based and synchrotron radiation based $\mu \mathrm{CT}$, Proc. SPIE (2008).

[2] P.J. Withers, X-ray nanotomography, Mater. Today 12 (2007) 26-34.

[3] V. Cosslett, W. Nixon, X-ray shadow microscopy, Nature 170 (1952) 436-438.

[4] Y.S. Touloukian, R.W. Powell, C.Y. Ho, P.G. Klemens, Thermal conductivity - metallic elements and alloys, Thermophysical Properties of Matter, 1970.

[5] J.E. Graebner, J.A. Herb, Dominance of intrinsic phonon scattering in intrinsic CVD diamond, Diamond Films Technol. 1 (1992) 155.

[6] J.E. Graebner, S. Jin, G.W. Kammlott, J.A. Herb, C.F. Gardinier, Large anisotropic thermal conductivity in synthetic diamond films, Nature 359 (1992) 401-440.

[7] J.E. Graebner, S. Jin, G.W. Kammlott, C.F. Gardinier, Unusually high thermal conductivity in diamond films, Appl. Phys. Lett. 60 (1992) 1576-1578.

[8] R.W. Powell, C.Y. Ho, P.E. Liley, Thermal conductivity of selected materials, NSRDSNBS 8 (1966).

[9] A. Muller, On the input limit of an X-Ray tube with circular focus, Proc. R. Soc. A (117) (1927) 30-42.

[10] J. Vine, P.A. Einstein, Heating effect of an electron beam impinging on a solid surface, allowing for penetration, IEEE Proc. (111) (1964) 921-930.

[11] M.K. Bhattacharyya, D.T. Tuma, Heating of solid target by electron beams, IEEE Trans. Electron Devices (30) (1983) 602-608.

[12] D.A. Grider, P.K. Ausburn, Electron beam melting in microfocus X-ray tubes, J. Phys. D. Appl. Phys. (19) (1986) 228.
[13] D. Possa, J.A. Nogueira, Algun problemas de electromagnetismo envolvendo series infinitas, Rev. Bras. Ensino Fis. (25) (2003) 384-387.

[14] D.X. Macedo, I. Guedes, Potencial electrico para distribuicoes de cargas puntiformes : sobre a convergencia de series infinitas, Rev. Bras. Ensino Fis. 32 (2010) 3309.

[15] D.C. Stickler, The thermal response of a metal slab to a class of radially dependent heat input, Appl. Res. Sci. A (14) (1965) 279-293.

[16] K. Kanaya, S. Okanaya, Penetration and energy loss theory of electrons in solid target, J. Phys. D. Appl. Phys. (5) (1972) 43.

[17] G.D. Archard, Backscattering of electrons, J. Appl. Phys. (32) (1961) 1505-1509.

[18] E.T. Swartz, R.O. Pohl, Thermal boundary resistance, Rev. Mod. Phys. 61 (3) (1989) 605-668.

[19] C. Monachon, L. Weber, Thermal boundary conductance of transition metals on diamond, Emerg. Mater. Res. 1 (2012).

[20] C. Monachon, L. Weber, Thermal boundary conductance between refractory metal carbides and diamond, Acta Mater. (73) (2014) 337-346.

[21] T.I. Galinka, A. Klokov, A. Sharkov, V.S. Bagaev, E.E. Onishchenko, V.V. Zaitsev, V.G. Ral'chenko, V.A. Dravin, R.A. Khmel'nitskii, A.A. Gippius, Propagation of acoustic phonons across the interfaces in CdTe and Si/CVD-diamond and quasi-twodimensional phonon wind in $\mathrm{CdTe} / \mathrm{ZnTe}$ quantum wells, Phys. B Condens. Matter (2002) 243-246.

[22] A. Klokov, D. Aminev, A. Sharkov, V. Ral'chenko, T. Galkina, Thermal parameters of layers and interfaces in silicon-on-diamond structures, Phys. Solid State 50 (12) (2008) 2263-2269.

[23] H. Verhoeven, E. Boettger, A. Flöter, H. Reiß, R. Zachai, Thermal resistance and electrical insulation of thin low-temperature-deposited diamond films, Diam. Relat. Mater. 6 (2-4) (1997) 298-302.

[24] P. Vadasz, Analytical solution to nonlinear thermal diffusion: Kirchhoff versus ColeHopf transformations, J. Heat Transf. (132) (2010).

[25] E.V. Ivakin, A.V. Sukhodolov, V.G. Ralchenko, A.V. Vlassov, A.V. Khomich, Measurement of thermal conductivity of polycrystalline CVD diamond by laser-induced transient grating technique, Quantum Electron. 32 (4) (2002) 367.

[26] E.A. Burgemeister, Thermal conductivity of natural diamond between 320 and 450 K, Physica 93 (2) (1977) 165-179.

[27] D.G. Onn, A. Witek, Y.Z. Qiu, T.R. Anthony, W.F. Banholzer, Some aspects of the thermal conductivity of isotopically enriched diamond single crystals, Phys. Rev. Lett. (19) (1992).

[28] J.R. Olson, R.O. Pohl, J.W. Vandersande, A. Zoltan, T.R. Anthony, W.F. Banholzer, Thermal conductivity of diamond between 170 and $1200 \mathrm{~K}$ and the isotope effect, Phys. Rev. B 47 (22) (1993) 14,850-14,856.

[29] R. Berman, M. Martinez, The thermal conductivity of diamonds, Thermal Conductivity, 14, Springer 1976, pp. 3-10.

[30] J.W. Vandersande, C.B. Vining, A. Zoltan, Thermal conductivity of natural type IIa diapond between $500 \mathrm{~K}$ and $1250 \mathrm{~K}$, 2nd International Symposium on Diamond Materials, 1991.

[31] H.-K. Lyeo, D.G. Cahill, Thermal conductance of interfaces between highly dissimilar materials, Phys. Rev. B 73 (14) (2006) 144,301.

[32] H.S. Carslaw, J.C. Jaeger, Conduction of Heat in Solids, Oxford University Clarendon Press, 1959.

[33] Y. Menesguen, R. Kuselewicz, Thermal modeling of lare-area VCSELs under optical pumping, IEEE J. Quantum Electron. (41) (2005) 901-908.

[34] A.V. Inyushkin, A.N. Taldenkov, V.G. Ralchenko, V.I. Konov, A.V. Khomich, R.A. Khmel'nitskiĪ, Thermal conductivity of polycrystalline CVD diamond: experiment and theory, J. Exp. Theor. Phys. 107 (3) (2008) 462-472.

[35] A. Khounsary, W. Philips, Thermal, structural, and fabrication aspects of diamond windows for high power synchrotron X-ray beamlines, Proc. SPIE (1739) (1993). 